\title{
Investigation of effect of electrode polarity on electrochemistry and magnetohydrodynamics using tertiary current distribution in electroslag remelting process
}

\author{
E. Karimi-Sibaki ${ }^{1} \cdot$ A. Kharicha ${ }^{1} \cdot$ A. Vakhrushev ${ }^{1} \cdot M$. Wu$^{2} \cdot$ A. Ludwig ${ }^{2} \cdot$ J. Bohacek $^{3}$
}

Received: 15 July 2021 / Revised: 30 August 2021 / Accepted: 1 September 2021 / Published online: 6 December 2021

(c) The Author(s) 2021

\begin{abstract}
Transport phenomena including the electromagnetic, concentration of ions, flow, and thermal fields in the electroslag remelting (ESR) process made of slag, electrode, air, mold, and melt pool are computed considering tertiary current distribution. Nernst-Planck equations are solved in the bulk of slag, and faradaic reactions are regarded at the metal-slag interface. Aiming at exploring electrochemical effects on the behavior of the ESR process, the calculated field structures are compared with those obtained using the classical ohmic approach, namely, primary current distribution whereby variations in concentrations of ions and faradaic reactions are ignored. Also, the influence of the earth magnetic field on magnetohydrodynamics in the melt pool and slag is considered. The impact of the polarity of electrode, whether positive, also known as direct current reverse polarity (DCRP), or negative, as known as direct current straight polarity (DCSP), on the transport of oxygen to the ingot of ESR is investigated. The obtained modeling results enabled us to explain the experimental observation of higher oxygen content in DCSP than that of DCRP operated ESR process.
\end{abstract}

Keywords Electroslag remelting $\cdot$ Electrochemistry $\cdot$ Calcium fluoride $\cdot$ Calcium oxide $\cdot$ Ion transport $\cdot$ Electro-migration

\section{List of symbols}

$\boldsymbol{A} \quad$ Magnetic vector potential, $\mathrm{V} \mathrm{s} \mathrm{m}^{-1}$

$A_{\text {electrode }}$ Electrode area, $\mathrm{m}^{2}$

$B \quad$ Magnetic field, $\mathrm{T}$

$B_{\mathrm{e}} \quad$ Earth magnetic field, $\mathrm{T}$

$b \quad$ Tafel slope, $\mathrm{V}$

c Concentration of each ion, $\mathrm{mol} \mathrm{m}^{-3}$

$\mathrm{C}^{+} \quad$ Concentration of cations, $\mathrm{mol} \mathrm{m}^{-3}$

$C^{-} \quad$ Concentration of anions, $\mathrm{mol} \mathrm{m}^{-3}$

$D \quad$ Diffusion coefficient of each ion, $\mathrm{m}^{2} \mathrm{~s}^{-1}$

$\boldsymbol{E} \quad$ Electric field, $\mathrm{V} \mathrm{m}^{-1}$

F Faraday constant, $\mathrm{A} \mathrm{s} \mathrm{mol}^{-1}$

A. Kharicha

Abdellah.kharicha@unileoben.ac.at

1 Christian-Doppler Laboratory for Metallurgical Applications of Magnetohydrodynamics, Montanuniversitaet of Leoben, Leoben A-8700, Austria

2 Chair of Simulation and Modeling of Metallurgical Processes, Montanuniversitaet of Leoben, Leoben A-8700, Austria

3 Heat Transfer and Fluid Flow Laboratory, Faculty of Mechanical Engineering, Brno University of Technology, Technicka 2896/2, 61669 Brno, Czech Republic
$\boldsymbol{F}_{\mathrm{L}} \quad$ Volumetric Lorentz force, $\mathrm{N} \mathrm{m}^{-3}$

$F_{r} \quad$ Radial Lorentz force, $\mathrm{N} \mathrm{m}^{-3}$

$F_{x} \quad$ Axial Lorentz force, $\mathrm{N} \mathrm{m}^{-3}$

$F_{\theta} \quad$ Tangential Lorentz force, $\mathrm{N} \mathrm{m}^{-3}$

$F_{\mathrm{LP}} \quad$ Poloidal Lorentz force, $\mathrm{N} \mathrm{m}^{-3}$

$F_{\mathrm{L} \theta} \quad$ Toroidal Lorentz force, $\mathrm{N} \mathrm{m}^{-3}$

g Gravity constant, $\mathrm{m} \mathrm{s}^{-2}$

$h \quad$ Enthalpy, $\mathrm{J} \mathrm{kg}^{-1}$

I Imposed electrical current, A

j Electric current density, $\mathrm{A} \mathrm{m}^{-2}$

$j_{r} \quad$ Radial current density, $\mathrm{A} \mathrm{m}^{-2}$

$j_{0} \quad$ Exchange current density in Tafel equation, A $\mathrm{m}^{-2}$

Boltzmann constant, $\mathrm{m}^{2} \mathrm{~kg} \mathrm{~s}^{-2} \mathrm{~K}^{-1}$

$\begin{array}{ll}k & \text { Boltzmann constant, } n^{2} \text { Normalized amount of oxygen }\end{array}$

$N \quad$ Total flux of each ion, $\mathrm{mol} \mathrm{m}^{-2} \mathrm{~s}^{-1}$

$p \quad$ Pressure, $\mathrm{Pa}$

$Q_{\mathrm{j}} \quad$ Joule heating, $\mathrm{W} \mathrm{m} \mathrm{m}^{-3}$

$R \quad$ Universal gas constant, $\mathrm{J} \mathrm{K}^{-1} \mathrm{~mol}^{-1}$

$r_{i} \quad$ Ion radius, $\mathrm{m}$

$t \quad$ Time, $\mathrm{s}$

$T \quad$ Temperature, $\mathrm{K}$

$T_{0} \quad$ Reference temperature, $\mathrm{K}$

$T_{\mathrm{m}} \quad$ Melting temperature, $\mathrm{K}$ 


\begin{tabular}{|c|c|}
\hline $\boldsymbol{u}$ & Velocity vector, $\mathrm{m} \mathrm{s}^{-1}$ \\
\hline$\nabla \boldsymbol{u}^{\mathrm{T}}$ & Transpose of velocity gradient tensor, $\mathrm{s}^{-1}$ \\
\hline$u_{\mathrm{P}}$ & Poloidal velocity, $\mathrm{m} \mathrm{s}^{-1}$ \\
\hline$u_{r}$ & Radial velocity, $\mathrm{m} \mathrm{s}^{-1}$ \\
\hline$u_{x}$ & Axial velocity, $\mathrm{m} \mathrm{s}^{-1}$ \\
\hline$u_{\theta}$ & Toroidal velocity, $\mathrm{m} \mathrm{s}^{-1}$ \\
\hline$X$ & Dimensionless distance \\
\hline$z$ & Charge number of each ion \\
\hline$\beta$ & Thermal expansion coefficient, $\mathrm{K}^{-1}$ \\
\hline$\beta_{\mathrm{s}}$ & Slag thermal expansion coefficient, $\mathrm{K}^{-1}$ \\
\hline$\beta_{\mathrm{m}}$ & Metal thermal expansion coefficient, $\mathrm{K}^{-1}$ \\
\hline$\lambda$ & Thermal conductivity, $\mathrm{W} \mathrm{K}^{-1} \mathrm{~m}^{-1}$ \\
\hline$\lambda_{\mathrm{s}}$ & Slag thermal conductivity, $\mathrm{W} \mathrm{K}^{-1} \mathrm{~m}^{-1}$ \\
\hline$\lambda_{\mathrm{m}}$ & Metal thermal conductivity, $\mathrm{W} \mathrm{K}^{-1} \mathrm{~m}^{-1}$ \\
\hline$\rho$ & Density, $\mathrm{kg} \mathrm{m}^{-3}$ \\
\hline$\rho_{0}$ & Reference density, $\mathrm{kg} \mathrm{m}^{-3}$ \\
\hline$\rho_{\mathrm{s}}$ & Slag density, $\mathrm{kg} \mathrm{m}^{-3}$ \\
\hline$\rho_{\mathrm{m}}$ & Metal density, $\mathrm{kg} \mathrm{m}^{-3}$ \\
\hline 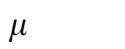 & Viscosity, $\mathrm{kg} \mathrm{s}^{-1} \mathrm{~m}^{-1}$ \\
\hline$u_{\mathrm{s}}$ & Slag viscosity, $\mathrm{kg} \mathrm{s}^{-1} \mathrm{~m}^{-1}$ \\
\hline$\mu_{\mathrm{m}}$ & Metal viscosity, $\mathrm{kg} \mathrm{s}^{-1} \mathrm{~m}^{-1}$ \\
\hline$\mu_{0}$ & Magnetic permeability, $\mathrm{H} \mathrm{m}^{-1}$ \\
\hline$\varphi$ & Electric potential, $\mathrm{V}$ \\
\hline$\eta$ & Overpotential, V \\
\hline & Electrical conductivity, $\mathrm{S} \mathrm{m}^{-1}$ \\
\hline$\sigma_{\text {Slag }}$ & Electrical conductivity of slag, $\mathrm{S} \mathrm{m}^{-1}$ \\
\hline$\sigma_{\text {Electrode }}$ & Electrical conductivity of electrode, $\mathrm{S} \mathrm{m}^{-1}$ \\
\hline$\sigma_{\text {Crucible }}$ & Electrical conductivity of crucible, $\mathrm{S} \mathrm{m}^{-1}$ \\
\hline$\sigma_{\text {Air }}$ & Electrical conductivity of air, $\mathrm{S} \mathrm{m}^{-1}$ \\
\hline & Divergence operator, $\mathrm{m}^{-1}$ \\
\hline & Gradient operator, $\mathrm{m}^{-1}$ \\
\hline
\end{tabular}

\section{Introduction}

Electroslag remelting (ESR) as a secondary metallurgical process is extensively used to purify metal and special alloys such as steel and nickel-based alloy. The impure metal/alloy electrode, immersed in a molten slag, is electrically heated through Joule heating. The electrode remelts to form droplets. They pass through the slag to reach the melt pool, where the solidification occurs in a water-cooled mold to build the clean, high-grade ingot [1]. The process comprises numerous phenomena, including heat transfer with phase change in the melting of the electrode or the solidification of ingot, the interaction among the flow and electromagnetic fields known as magnetohydrodynamics (MHD), and the mass transfer of chemical species.

Contributions of mass transfer in ESR are identified in the refinement of the alloy [2-4], removal and precipitation of nonmetallic inclusions in the slag and melt pool $[5,6]$, and chemical and electrochemical reactions in the slag
[7, 8]. Several electrochemical (faradaic) reactions take place at slag-metal or slag-mold interfaces to purify the alloy. The $\mathrm{CaF}_{2}$-based slag in ESR, typically made of $\mathrm{CaF}_{2}, \mathrm{CaO}$, and $\mathrm{Al}_{2} \mathrm{O}_{3}$, is inherently an electrolyte. Thereby, faradaic reactions of several elements, such as $\mathrm{Ti}$, $\mathrm{O}, \mathrm{S}, \mathrm{Fe}, \mathrm{Ca}$, and $\mathrm{Al}$, were reported [7]. The electrochemical transport of ions like $\mathrm{Ca}^{2+}, \mathrm{Al}^{3+}, \mathrm{Fe}^{2+}, \mathrm{F}^{-}$, and $\mathrm{O}^{2-}$ plays a pivotal role in the electrical behavior of the slag and consequently in the performance of the process [7-9]. Different contributions of electrochemistry during direct current (DC) operation of ESR were reported as follows: higher melting rate of the electrode with positive polarity as known as direct current reverse polarity (DCRP) than that with negative polarity as known as direct current straight polarity (DCSP) [10-12], higher oxygen content in the ingot of DCSP than that of DCRP [13, 14], higher oxygen content in the ingot using $\mathrm{CaF}_{2}-\mathrm{Al}_{2} \mathrm{O}_{3}$ slag than that using $\mathrm{CaF}_{2}-\mathrm{CaO}$ [15], and formation of a thick layer of iron oxide $(\mathrm{FeO})$ under the tip of the electrode in DCRPoperated ESR [14, 16].

The thermodynamic and kinetic models were suggested to study chemical and electrochemical reactions in ESR [17-19] considering primary current distribution, whereby the electrical resistance of the slag is determined through Ohm's law. Customarily, primary, secondary, and tertiary current distributions were introduced to study electrochemical systems [20]. Ohm's law is also applied in secondary current distribution, where the effect of faradaic reaction of the reactant (electrode kinetics) is considered assuming a uniform concentration field of all involving ions in the electrolyte [20,21]. Tertiary current distribution accounts for both electrode kinetics and the non-uniformity of ion concentration field. The electrolyte behaves in accordance with Nernst-Planck equations subject to the approximation of electro-neutrality. The slag in ESR is an electrolyte where the field structures, such as electromagnetic, concentration of ions, thermal, and flow fields, are greatly influenced by the distribution of electric current density.

Herein, we propose a model considering tertiary current distribution in the ESR process. Transport phenomena in an ESR system made of the electrode, slag (electrolyte), air, melt pool, and mold are calculated. The computational domain is shown in Fig. 1. The electromagnetic field is calculated in the entire system. The thermal field is calculated in the slag, mold, and melt pool, whereas the flow is modeled in the slag and melt pool. Distributions of concentration of ions in the bulk of slag, $\mathrm{CaF}_{2}-25$ mass\% $\mathrm{CaO}$, are computed. Faradaic reactions at the metal-slag interface are modeled following Tafel law [20, 22]. Another calculation is performed considering the widely used classical ohmic approach $(\Omega)$ (primary current distribution) in ESR [23]. A comparison is made between the 


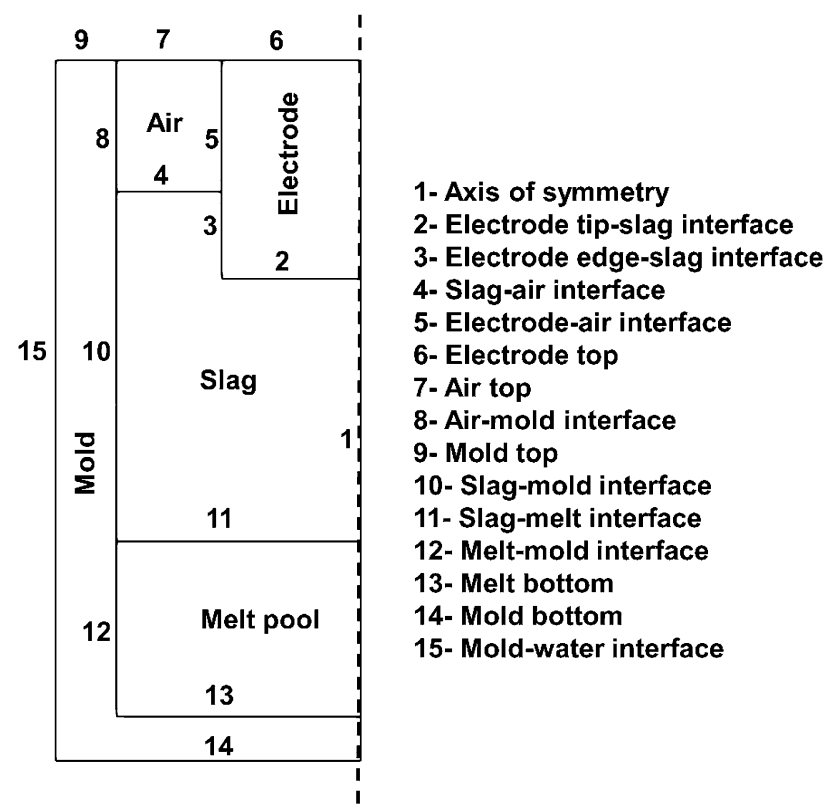

Fig. 1 Configuration of computational domain and boundaries

obtained results through the classical approach and the presented model in this study to explore the impact of electrochemical effects on field structures in the ESR process. Based on our modeling results, we put forward an explanation for the phenomenon, namely higher oxygen content in the ingot of DCSP than that of DCRP [13, 14], in the ESR process.

\section{Modeling}

All symbols used in this paper are described in "List of symbols". All governing equations, Eqs. (1) through (13), related to the flow, thermal, electromagnetic, and concentration of ion fields are listed in Table 1, and they will be further described in this section.

For the sake of simplicity and to avoid extra complexity, several assumptions are made as follows:

(i) The movement of slag-melt and slag-air interfaces is not modeled so that those interfaces remain stationary.

(ii) The solidification of the metal in the melt pool as well as remelting of the electrode and consequently dripping droplets through the slag is not taken into account in the model.

(iii) Chemical reactions like generation/recombination that may occur in the bulk of molten slag are not included in the model. The electrolyte (molten slags) is assumed to be entirely dissociated at the elevated temperature of the process $(>2000 \mathrm{~K})$. Therefore, all molecules of the slags split into ions as follows:
Table 1 Governing equations of flow, magnetic, concentration of ions, and electric current density fields

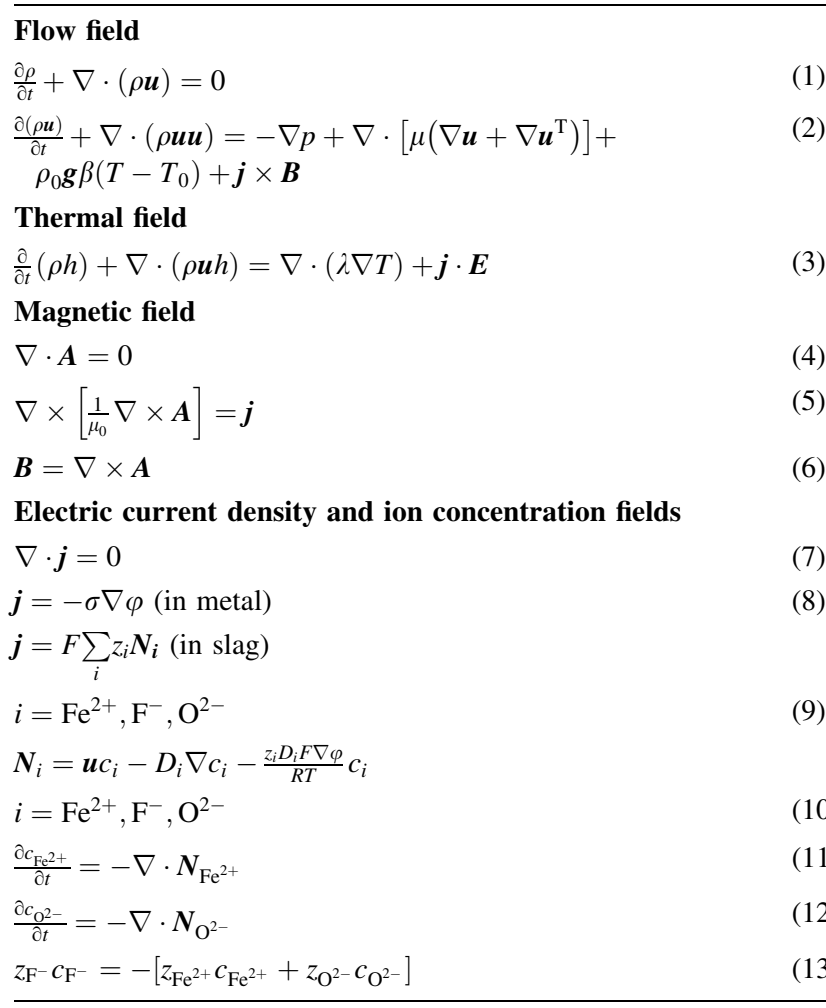

$\mathrm{CaO} \rightarrow \mathrm{Ca}^{2+}+\mathrm{O}^{2-}$

(iv) Both $\mathrm{Ca}^{2+}$ and $\mathrm{Fe}^{2+}$ can participate in faradaic reactions at the cathode. In fact, $\mathrm{Ca}^{2+}$ and $\mathrm{Fe}^{2+}$ compete to gain an electron at the cathode. The standard reduction potential and consequently the priority of gaining electron are higher for $\mathrm{Fe}^{2+}$ than that of $\mathrm{Ca}^{2+}$ [20]. Herein, we ignore this priority; thus, any available cation $\left(\mathrm{Fe}^{2+}\right.$ or $\mathrm{Ca}^{2+}$ ) reacts at the surface of the cathode. This assumption helps us to simplify the problem further. The electric current delivered from/to slag to/from metal at cathode/anode can be modeled considering only one of the cations. Herein, only faradaic reactions related to iron are included in the model as follows: oxidation of iron at the anode $\left(\mathrm{Fe} \rightarrow \mathrm{Fe}^{2+}+2 \mathrm{e}\right)$ and reduction of iron at the cathode $\left(\mathrm{Fe}^{2+}+2 \mathrm{e} \rightarrow \mathrm{Fe}\right)$. Correspondingly, it is also sufficient to solve the transport equation of one cation (here $\mathrm{Fe}^{2+}$ ) considering that the initial concentration of $\mathrm{Fe}^{2+}$ is equivalent to that of $\mathrm{Ca}^{2+}$. Additionally, transport equations of anions $\left(\mathrm{F}^{-}, \mathrm{O}^{2-}\right)$ must be solved to calculate the electric current density field in the bulk of slag. The latter 
will be further elucidated after introducing governing equations related to the concentration of ions in Sect. 2.1.

(v) The formation of cathodic/anodic electric double layer results in a potential drop at the metal-slag interface that is implicitly modeled through Tafel equation: $\boldsymbol{j}=j_{0} \mathrm{e}^{-\frac{\eta}{b}}$ [20]. The required parameters for Tafel equation, which describes the relationship between the overpotential/potential jump and electric current density, as shown in Fig. 2a, at the anode and cathode were extracted from Ref. [16]. The potential jump $(<0.1 \mathrm{~V})$ aids to make a relatively uniform distribution of the electric current density along the metal-slag interface, although that is negligible compared to the total applied potential $(\sim 20 \mathrm{~V})$ in the process.

(vi) Diffusion coefficient has a decisive impact on the transport of ion. It is dependent on several parameters such as temperature, pressure, electrolyte composition, radius, and valency of ion [24]. As recommended in Ref. [24] for ESR slags, we use Eyring relationship $\left(D=\frac{k T}{2 r_{i} \mu_{\mathrm{s}}}\right)$ to obtain the diffusion coefficient as a function of slag temperature, slag viscosity, and ion radius. Of note, the viscosity of the slag in this study is also dependent on temperature $\left(\mu_{\mathrm{s}}=9.217 \times 10^{5} \mathrm{e}^{-0.00878 T}\right) \quad$ [24]. Indicatively, diffusion coefficients of involving ions are plotted against temperature in Fig. 2b.

(vii) Any plausible electronic conduction within the bulk of slag is ignored [25]. Thus, the electric current is carried only by the movement of ions.

(viii) The solutal buoyancy force originated in the spatial variation in the ion concentration field is ignored. However, the thermal buoyancy force and Lorentz force originated in the interaction between the electric current density and the self-induced magnetic field are taken into account.

(a)

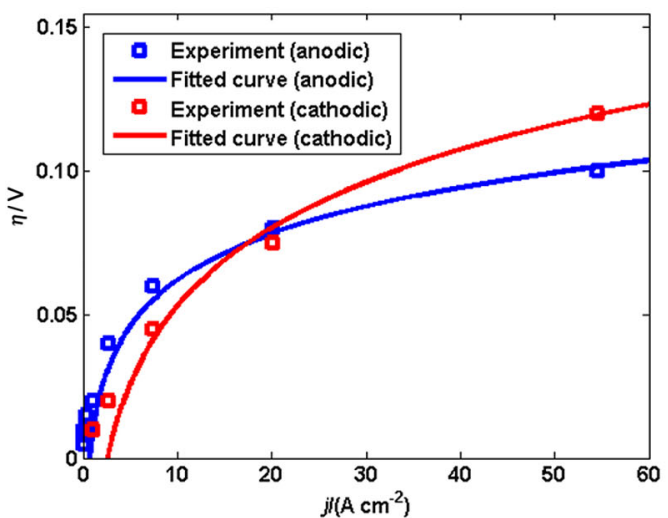

(ix) The bulk of slag always remains electrically neutral [20]. The bulk electro-neutrality condition is mathematically expressed as: $\sum_{i} z_{i} c_{i}=0, i=\mathrm{Fe}^{2+}, \mathrm{F}^{-}, \mathrm{O}^{2-}$.

(x) In the present study, the numerical model is configured based on experimental measurements of Mitchell and Beynon [16]. The experiment was conducted using a particular mold in which the inner surface was painted by boron-nitride to ensure that no electric current flows through the mold at slag-mold and melt-mold interfaces. Thus, the electric current was only collected from the base plate. Following their experiment, no mold current is considered in our model.

The induced magnetic field is dominantly in the azimuthal direction. Herein, the influence of the earth magnetic field on the flow is also considered. Therefore, a 2D axisymmetric swirl model is applied on a $2 \mathrm{D}$ axisymmetric computational domain which is illustrated in Fig. 1.

\subsection{Governing equations}

All governing equations to calculate transport phenomena are listed in Table 1. Equations of continuity, Eq. (1), and momentum, Eq. (2), are solved to determine the flow field. Source terms in momentum equation are Lorentz force $\left(\boldsymbol{F}_{\mathrm{L}}=\boldsymbol{j} \times \boldsymbol{B}\right)$ and thermal buoyancy force, which is calculated considering Boussinesq approximation. The interaction between the earth magnetic field and the radial component of current density creates the Lorentz force in azimuthal direction [26] that is included in our model $\left(F_{\mathrm{L} \theta}=j_{r} B_{\mathrm{e}}\right)$.

No-slip boundary condition is used at all boundaries except the slag-air interface where the free-slip condition is assigned. The flow in the air zone is not calculated.

The thermal field is calculated considering the enthalpy conservation equation through Eq. (3). The source term in

(b)

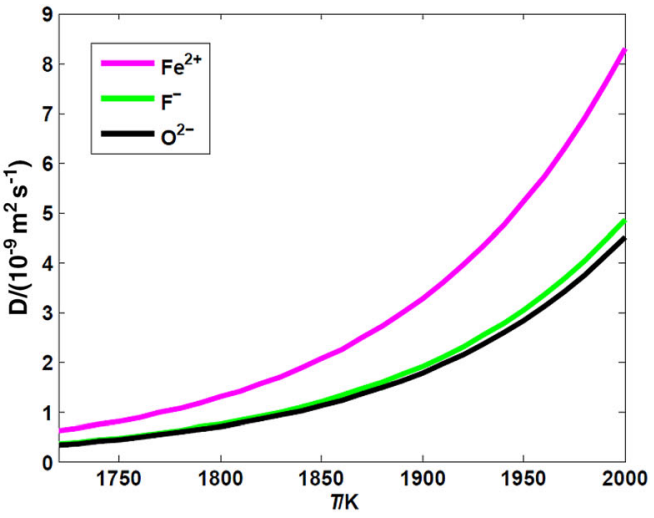

Fig. 2 Fitting curves of total overpotential against amount of imposed electric current density (a) and diffusion coefficients of involving ions against temperature considering Eyring relationship (b) 
Eq. (3) is Joule heating $\left(Q_{\mathrm{j}}=\boldsymbol{j} \cdot \boldsymbol{E}\right)$. Common thermal boundary conditions are assigned as follows [23, 27, 28]. The radiation boundary condition with the emissivity of 0.8 is considered at the slag-air interface. The melting temperature of the electrode $(1811 \mathrm{~K})$ made of pure iron is assigned at electrode tip-slag and electrode edge-slag interfaces. The liquidus temperature of slag $(1725 \mathrm{~K})$ is set at following boundaries on the slag/melt side: slag-mold, melt-mold, and mold bottom. They are conjugate walls where the slag skin is implicitly modeled considering a constant thickness $(\sim 1 \mathrm{~mm})$. Thus, the heat transfer coefficient of $500 \mathrm{~W} \mathrm{~m}^{-2} \mathrm{~K}^{-1}$ is assumed at slag-mold, melt-mold, and mold bottom on the mold side. The heat transfer coefficient at the mold bottom and the mold-water interface is assigned to $7000 \mathrm{~W} \mathrm{~m}^{-2} \mathrm{~K}^{-1}$. Furthermore, the two sides of the wall for the slag-melt interface are thermally coupled. The thermal field is not calculated in the air and electrode zones.

The $\boldsymbol{A}-\varphi$ formulation, Eqs. (5) and (6), considering Coulomb gauge, Eq. (4), is utilized to determine the electromagnetic field [28-30]. The magnetic permeability is assumed to remain constant in the entire domain. Magnitudes of radial and axial components of magnetic vector potential are set zero at mold bottom and mold-water interface. The flux of magnetic vector potential is zero at the electrode top, air top, and mold top. Continuity of magnetic vector potential is considered at all interior boundaries.

Ohm's law is applied in the entire domain, including air, electrode, melt pool, and mold, whereas Nernst-Planck equations, Eq. (9) through Eq. (13) as listed in Table 1, are utilized to determine electric current density field in the slag considering tertiary current distribution [31]. The conservation of electric current density, Eq. (7), is solved. The electric current density in the slag is obtained using the total mass flux of all involving ions through Eq. (9). The total mass flux of each ion, Eq. (10), including advection flux, diffusion flux, and electro-migration flux, is taken into account. The total flux of each ion must be conserved as described mathematically by Eqs. (11) and (12). The imposition of the bulk electro-neutrality as described in assumption (ix) helps us to calculate the concentration field of the non-reacting $\mathrm{F}^{-}$through Eq. (13). Of note, aiming at comparing the results with the classical ohmic approach extensively used to calculate electric current density in the slag [23], one calculation is also performed considering Ohm's law in the slag.

Boundary conditions of electric potential and concentration of ions are interdependent [31]. The total mass flux of non-reacting ions $\left(\mathrm{F}^{-}, \mathrm{O}^{2-}\right)$ is zero at all boundaries enclosing the slag. The mass flux of ferrous ion $\left(\mathrm{Fe}^{2+}\right)$ is assigned at the electrode tip-slag, electrode edge-slag, and slag-melt interfaces as follows: $\boldsymbol{j}= \pm z_{\mathrm{Fe}^{2+}} F N_{\mathrm{Fe}^{2+}}$. The positive sign is used for the anodic boundary, and the negative sign is used for the cathodic boundary. The mold current is ignored as described in assumption (x) so that the mass flux of ferrous ion is set to zero at the slag-mold interface.

The magnitude of electric potential at the top of the mold is zero. The flux of electric potential (electric current density) at electrode top is specified $\left(j= \pm \frac{I}{A_{\text {electrode }}}\right)$ to ensure an equal amount of electric current density flows through the system regardless of the condition of the slag electrical conductivity. The positive sign is used if the electrode is anodic, and the negative sign is used if the electrode is cathodic. The electric potential at the interface between slag and anode/cathode is assigned using Tafel law as described in assumption (v) to account for the electric double layer formation and, consequently, the overpotential [31]. The relationship between overpotential and current density is plotted in Fig. 2a. Simple regression analysis helps us to obtain the parameters of Tafel law. The experimental measurements are reproduced from Ref. [16]. Anodic and cathodic boundaries are electrode tip-slag, electrode edge-slag, and slag-melt interfaces. At all other interior boundaries, the continuity of the electric field is assigned.

\subsection{Other settings}

Mitchell and Beynon [16] conducted a series of experiments in an ESR apparatus to measure the overpotential and the amount of transferred oxygen to the ingot. The 2D axisymmetric swirl model is configured based on their study [16]. The computational domain including boundaries and regions is shown in Fig. 1. The electrode with the diameter of $3.18 \mathrm{~cm}$ was immersed with a depth of $1 \mathrm{~cm}$ into the slag made of $\mathrm{CaF}_{2}-25$ mass $\% \mathrm{CaO}$. The length of the slag cap on the ingot was $4 \mathrm{~cm}$. The electrode was made of iron, and subsequently, an ingot made of iron with the diameter of $5.5 \mathrm{~cm}$ was produced. Only $2 \mathrm{~cm}$ of the melt pool at the top of the ingot is included in the model to ensure that the full coupling of phenomena at the slag-melt interface is calculated. Details of the experiment are described in Ref. [16]. A very fine mesh with a total of 0.2 million volume elements was generated. We used the commercial software, FLUENT-ANSYS v. 14.5, to implement modeling equations with the help of user-defined functions. The software utilizes the finite volume method (FVM) to precisely model transport phenomena [32]. Within the framework of FVM, mass conservations of all ions are automatically satisfied. Considering the pseudotransient computation technique, transient calculations are made to obtain steady-state results, subject to further 
evaluations. All parameters used in our calculations are listed in Table 2.

\section{Results}

\subsection{Electromagnetic field}

The distribution of electric current density and consequently the self-induced magnetic field creates the Lorentz force that drives the flow in both the slag and melt. As shown in Fig. 3, a comparison considering the computed electric current density and magnetic fields is made between processes under different operating conditions, such as electrode anodic $(+)$, electrode cathodic $(-)$, and the classical ohmic approach $(\Omega)$. For the latter, the

Table 2 Parameters used in our calculations

\begin{tabular}{|c|c|c|}
\hline Parameter & Unit & Value \\
\hline$\mu_{0}$ & $\mathrm{H} \mathrm{m}^{-1}$ & $4 \pi \times 10^{-7}$ \\
\hline$R$ & $\mathrm{~J} \mathrm{~K}^{-1} \mathrm{~mol}^{-1}$ & 8.314546 \\
\hline$g$ & $\mathrm{~m} \mathrm{~s}^{-2}$ & 9.81 \\
\hline$F$ & A s mol ${ }^{-1}$ & 96,485 \\
\hline$k$ & $\mathrm{~m}^{2} \mathrm{~kg} \mathrm{~s}^{-2} \mathrm{~K}^{-1}$ & $1.38 \times 10^{-23}$ \\
\hline$I$ & A & 991 \\
\hline$r_{\mathrm{Fe}^{2+}}$ & $\mathrm{m}$ & $0.76 \times 10^{-10}$ \\
\hline$r_{\mathrm{F}^{-}}$ & $\mathrm{m}$ & $0.13 \times 10^{-9}$ \\
\hline$r_{\mathrm{O}^{2-}}$ & $\mathrm{m}$ & $0.14 \times 10^{-9}$ \\
\hline$T_{\mathrm{m}}$ & $\mathrm{K}$ & 1811 \\
\hline$B_{\mathrm{e}}$ & $\mathrm{T}$ & $5 \times 10^{-5}$ \\
\hline$\sigma_{\text {Electrode }}$ & $\mathrm{S} \mathrm{m}^{-1}$ & $8 \times 10^{5}$ \\
\hline$\sigma_{\text {Crucible }}$ & $\mathrm{S} \mathrm{m}^{-1}$ & $4 \times 10^{7}$ \\
\hline$\sigma_{\mathrm{Air}}$ & $\mathrm{S} \mathrm{m}^{-1}$ & $10^{-10}$ \\
\hline$\sigma_{\text {Slag }}$ & $\mathrm{S} \mathrm{m}^{-1}$ & Calculated \\
\hline$\rho_{\mathrm{s}}$ & $\mathrm{kg} \mathrm{m}^{-3}$ & 2650 \\
\hline$\mu_{\mathrm{s}}$ & $\mathrm{kg} \mathrm{s}^{-1} \mathrm{~m}^{-1}$ & Calculated \\
\hline$\rho_{\mathrm{m}}$ & $\mathrm{kg} \mathrm{m}^{-3}$ & 6980 \\
\hline$\mu_{\mathrm{m}}$ & $\mathrm{kg} \mathrm{s}^{-1} \mathrm{~m}^{-1}$ & 0.006 \\
\hline$\beta_{\mathrm{s}}$ & $\mathrm{K}^{-1}$ & 0.00005 \\
\hline$\beta_{\mathrm{m}}$ & $\mathrm{K}^{-1}$ & 0.00014 \\
\hline$\lambda_{\mathrm{s}}$ & $\mathrm{W} \mathrm{K} \mathrm{K}^{-1} \mathrm{~m}^{-1}$ & 10 \\
\hline$\lambda_{\mathrm{m}}$ & $\mathrm{W} \mathrm{K} \mathrm{K}^{-1} \mathrm{~m}^{-1}$ & 35 \\
\hline$j_{0}$ & $\mathrm{~A} \mathrm{~m}^{-2}$ & 8600 and 8940 \\
\hline$b$ & V & 0.044 and 0.087 \\
\hline$z_{\mathrm{Fe}^{2+}}$ & - & +2 \\
\hline$z_{\mathrm{F}^{-}}$ & - & -1 \\
\hline$z_{\mathrm{O}^{2-}}$ & - & -2 \\
\hline$D_{i}\left(i=\mathrm{Fe}^{2+}, \mathrm{F}^{-}, \mathrm{O}^{2-}\right)$ & $\mathrm{m}^{2} \mathrm{~s}^{-1}$ & Calculated \\
\hline
\end{tabular}

(a)

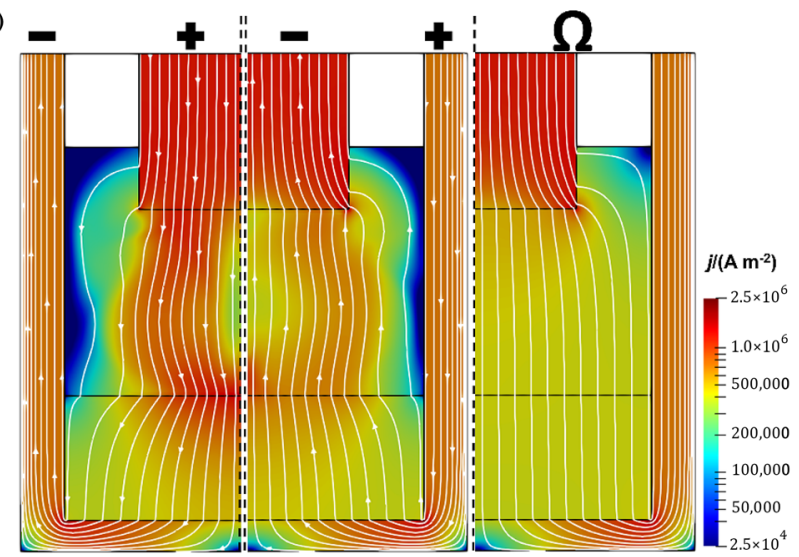

(b)

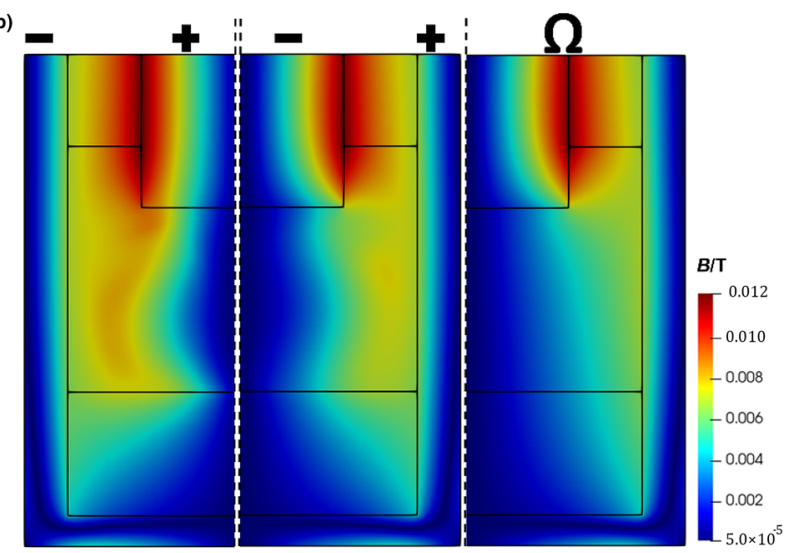

Fig. 3 Magnitude and streamlines of electric current density (a) and magnetic field (b)

slag electrical conductivity $\left(550 \mathrm{~S} \mathrm{~m}^{-1}\right)$ is independent of thermal and concentration fields. Thus, the electrochemical effects on the field structures are ignored. On the other hand, the electrochemical effects significantly influence the field structures, such as the electric current density field as shown in Fig. 3a and the magnetic field as shown in Fig. 3b. As described in assumption (v), no mold current is considered. The direction of the electric current is always from the anode to the cathode, as shown in Fig. 3a. Of note, the direction is undistinguished in the ohmic approach as electrode polarity is ignored. The peak of electric current density is observed under the shadow of the electrode when the electrode is anodic. In contrast, the peak is observed on the edge of the electrode when the electrode is cathodic or when ignoring the electrochemical effects for the ohmic approach. The intensity of electric current density decreases from the slag/metal bulk center toward the mold. The peak value of the magnetic field exists on the lateral wall of the electrode, as shown in Fig. 3b. A region of an intense magnetic field within the bulk of slag under the shadow of the electrode is also observed when the process runs considering an anodic electrode. 


\subsection{Velocity field}

The distribution of Lorentz force field, $\boldsymbol{F}=\left(F_{r}, F_{\theta}, F_{x}\right)$, governs the velocity field. The strength of Lorentz force in the melt pool is determined by the interplay between the electric current density and the magnetic fields, including the self-induced and earth magnetic fields. As shown in Fig. $4 \mathrm{a}$, an intense Lorentz force exists in the poloidal direction $\left(F_{\mathrm{LP}}=\sqrt{F_{r}^{2}+F_{x}^{2}}\right)$ under the shadow of electrode when the electrode is anodic. Therein, both magnetic field and electric current density are strong. Lorentz force is globally directed toward the center of the ingot in the poloidal plane. Unlike the calculated distribution of force for the classical/ohmic approach, the force bends downward adjacent to the slag-melt interface considering electrochemical effects. The interaction between the earth magnetic field and the radial component of electric current density gives rise to the toroidal part of Lorentz force $\left(F_{\theta}=F_{\mathrm{L} \theta}\right)$ as shown in Fig. $4 \mathrm{~b}$. The peak value is observed near the edge of electrode. In the bulk of slag and melt, the toroidal Lorentz force is minimal due to the lack of radial current density when the ohmic/classical approach is considered. In contrast, a notable amount of toroidal Lorentz force near the slag-melt interface, where the electric current density bends, is observed considering electrochemical effects.

The distribution of velocity force field, $\boldsymbol{u}=\left(u_{r}, u_{\theta}, u_{x}\right)$, is analyzed considering the poloidal velocity $\left(u_{\mathrm{P}}=\sqrt{u_{r}^{2}+u_{x}^{2}}\right)$ and toroidal velocity $\left(u_{\theta}\right)$. Regardless of the polarity of the electrode, an intense electro-vortex flow with the peak value in the bulk of slag under the shadow of the electrode in the poloidal plane is predicted. The ohmic approach also predicts a relatively similar electro-vortex flow pattern, as illustrated in Fig. 4c. Note that equisized vectors are used to indicate the direction. Surprisingly, the presence of a weak axial magnetic field such as the one from earth $\left(5 \times 10^{-5} \mathrm{~T}\right)$ gives rise to a flow in the toroidal direction. As shown in Fig. 4d, the flow pattern considering the ohmic approach differs from the pattern considering the electrochemical effects. The positive value means that the vector points inward, whereas the negative value means that the vector points outward in the toroidal plane The classical approach predicts an intense toroidal flow under the shadow of the electrode and a weak toroidal flow in the bulk of the melt pool. Conversely, a strong toroidal flow exists in the melt pool considering electrochemical effects.

\subsection{Thermal field}

The Joule heating $(\boldsymbol{j} \cdot \boldsymbol{E})$ as described in Eq. (3) (see Table 1), is supplied to heat the slag of the process. Accordingly, the electric current density field has a
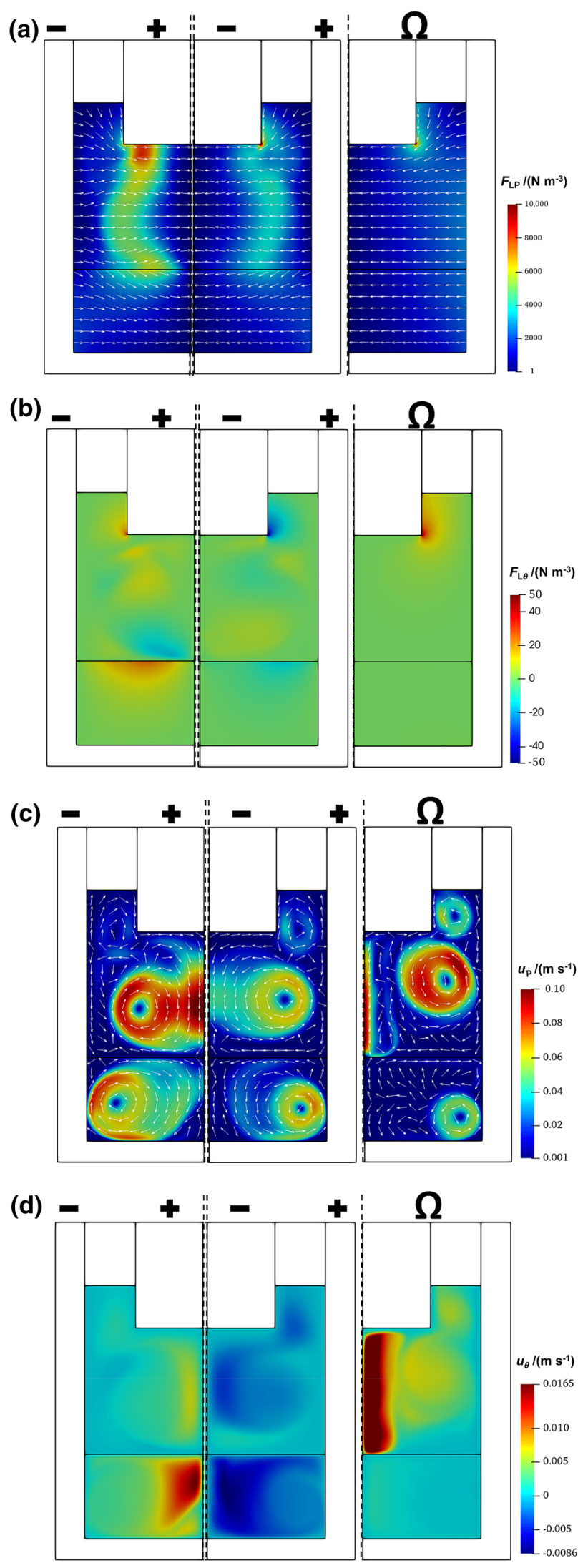

Fig. 4 Poloidal Lorentz force (a), toroidal Lorentz force (b), poloidal velocity $(\mathbf{c})$, and toroidal velocity $(\mathbf{d})$ 
decisive role in the distribution of Joule heating. Assuming ohmic conduction in the slag, the maximum amount of Joule heating is released near the edge of the electrode, as illustrated in Fig. 5a. On the other hand, a remarkable amount of Joule heating is observed in the vicinity of the slag-melt interface considering electrochemical effects. Of note, the amount of Joule heating is negligible in the electrode, ingot, and mold due to the low electrical resistance of the metal. As shown in Fig. 5b, the region where the flow recirculates matches the hottest area in the slag zone. A substantial part of generated heat in the bulk of slag is transferred through the mold wall to the cooling water. Additionally, a small amount of electric current flows adjacent to the mold wall to generate Joule heating. Thus, the temperature notably decreases from the bulk of slag toward the region near the mold wall.

\subsection{Concentration field}

The ensemble behavior of cations and anions is discussed in this section. The only cation considered in this study is ferrous ion $\left(\mathrm{Fe}^{2+}\right)$, and anions are $\mathrm{F}^{-}$and $\mathrm{O}^{2-}$. The cation concentration field, including the charge number $\left(2 \mathrm{Fe}^{2+}\right)$ is shown in Fig. 6a. It is anticipated that cations always

(a)

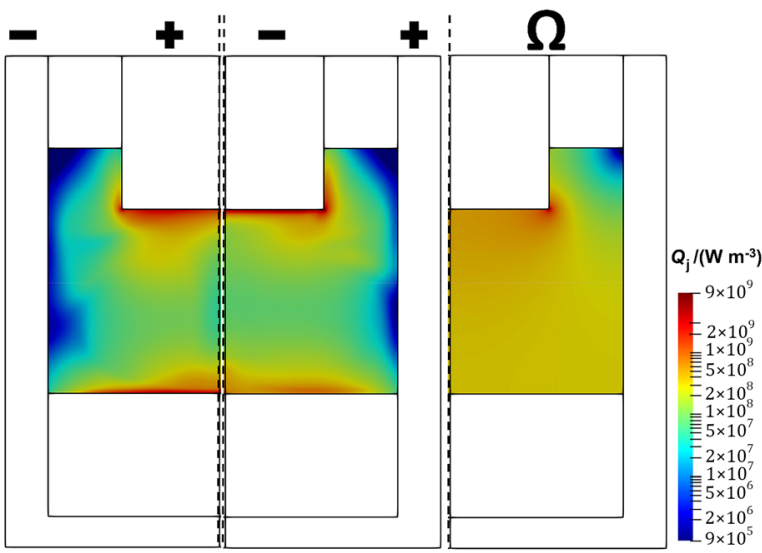

(b)

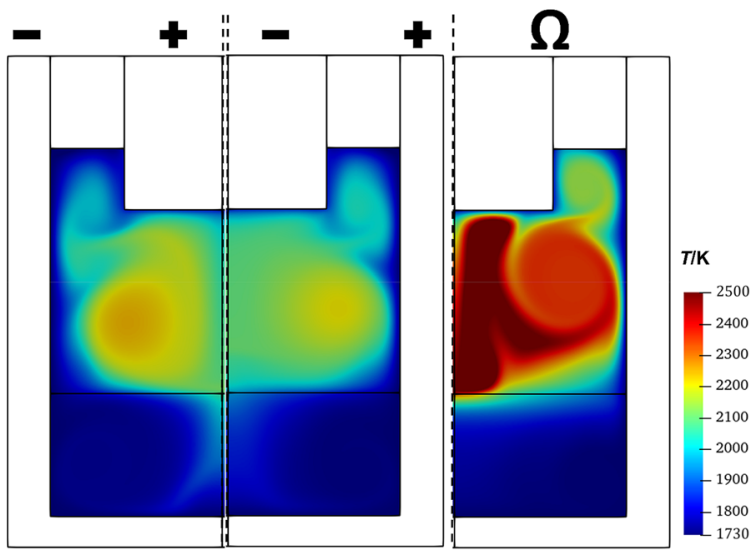

Fig. 5 Joule heating in slag zone (a) and temperature field (b) accumulate near the cathode. Contrastingly, a high concentration of the cation is near the electrode when the electrode is anodic, whereas cation piles up near the slagmelt interface when the electrode is cathodic. The electric current is delivered from/to slag to/from metal by faradaic reaction of ferrous ion. Consequently, an enormous amount of ferrous ion is injected into the slag at the electrode-slag interface for the anodic electrode or at the slag-melt interface for the cathodic electrode. In other words, the faradic reaction of ferrous ion determines the concentration field of cations in the ESR process. On the other hand, the absolute value of the concentration field of anions $\left(-\mathrm{F}^{-}-2 \mathrm{O}^{2-}\right)$ is governed by electro-migration of anions, as shown in Fig. 6b. Therefore, anions accumulate near the anodic electrode, whereas they move toward the slag-melt interface when the electrode is cathodic. The electrical conductivity of the slag considering electrochemical effects is dependent on the concentration of ions and thermal fields, $\sigma_{\text {Slag }}=\sum_{i} \frac{z_{i}^{2} D_{i} F^{2}}{R T} c_{i}$, whereas a uniform concentration field and a uniform electrical conductivity are assumed in the ohmic approach [20]. Apparently, a non-uniform electrical conductivity is predicted in the slag considering electrochemistry, as shown in Fig. 6c. The peak value of electrical conductivity is observed in the region where the flow recirculates at elevated temperature. Despite a relatively uniform concentration of ions in the bulk of slag, especially near the vortex, the temperature and consequently the diffusion coefficients of ions are significant.

The total applied potential of $20 \mathrm{~V}$ was reported during the experiment from the apparatus designed by Mitchell and Beynon [16]. However, the predicted potential drop in the classical approach $(\sim 24 \mathrm{~V})$, anodic electrode $(\sim 12 \mathrm{~V})$, and cathodic electrode $(\sim 14 \mathrm{~V})$ differs from the reported experimental value. Possible sources of the discrepancy are ignoring the movement of interfaces, assumption (i), ignoring the incomplete dissociation of molecules to ions, assumption (iii), and uncertainties on the experimental setup such as the shape of the electrode tip, the immersion depth of electrode, and the height/volume of the slag cap. Nevertheless, the variations in the calculated potential drop are associated with dissimilarities in the calculated electrical conductivity of the slag, as shown in Fig. 6c.

\section{Discussion}

Mitchell and Beynon [16] designed the laboratory scale ESR process, as shown in Fig. 1, to study the influence of electrochemical reactions at the metal-slag interface and to explore the refining mechanisms. In the experiment, the anodic electrode is tantamount to the DCRP condition, 
(a)

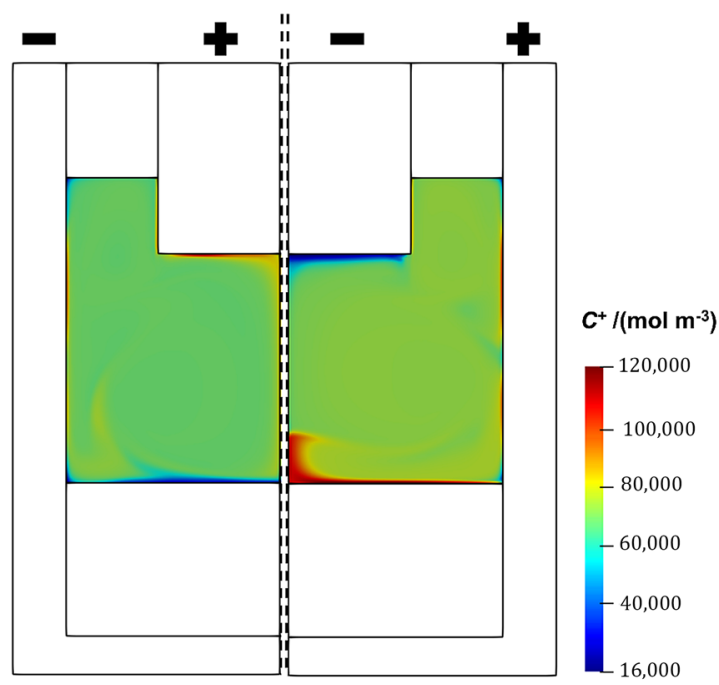

(b)

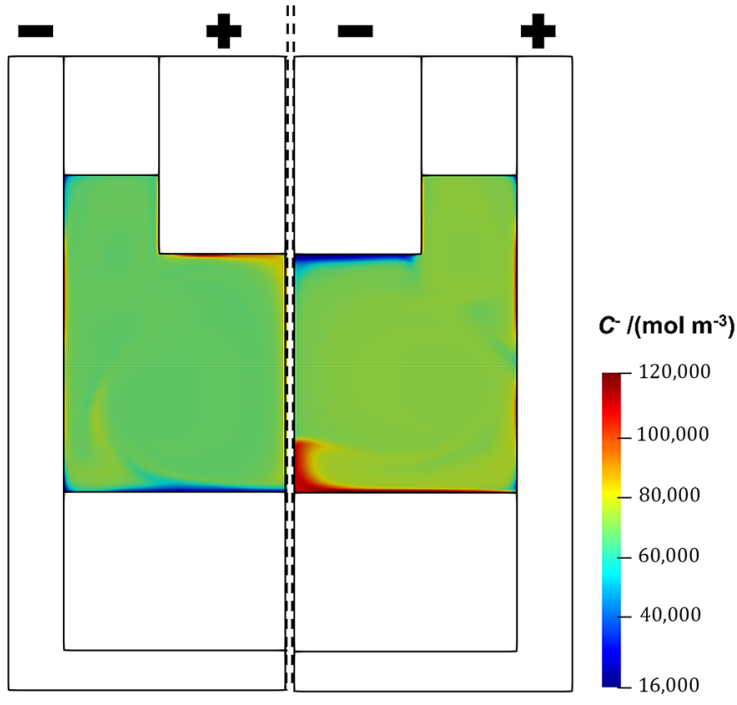

(c)

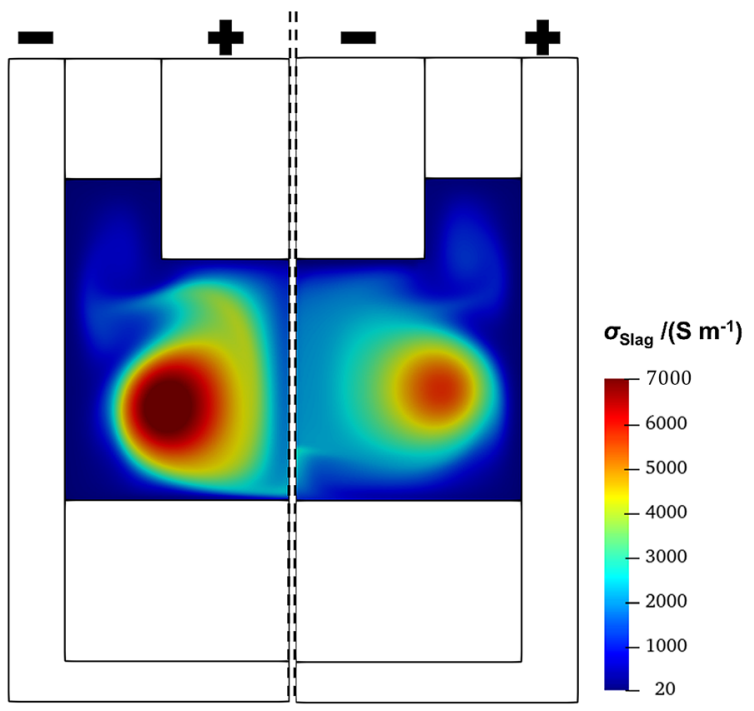

Fig. 6 Concentration of cations (a), concentration of anions (b) and electrical conductivity (c) while the cathodic electrode represents the DCSP condition. They reported a higher oxygen content in the final ingot using DCSP than that using DCRP [16]. This observation was numerously reported during the operation of the DC electroslag remelting process [13-16].

The calculated concentration field of oxygen is illustrated in Fig. 7a. As anticipated, oxygen as an anion migrates toward the anode. Thereby, the concentration of oxygen near the ingot top surface is higher in DCSP than that of DCRP. The oxygen is afterward provided to the ingot at the slag-melt interface through the discharge reaction as follows: $\mathrm{O}^{2-}-2 \mathrm{e} \rightarrow[\mathrm{O}]$. To identify the amount of final oxygen in the ingot, the oxygen concentration along the slag-melt interface is plotted as shown in Fig. $7 \mathrm{~b}$. Scales for normalization are the concentration of the oxygen in the bulk of $\mathrm{CaF}_{2}-25$ mass\% $\mathrm{CaO}$ slag on the $y$-axis and the radius of ingot on the $x$-axis. The available oxygen on the interface is precipitated as inclusions in the ingot. Evidently, the oxygen concentration along the interface using DCSP is approximately one order of

(a)

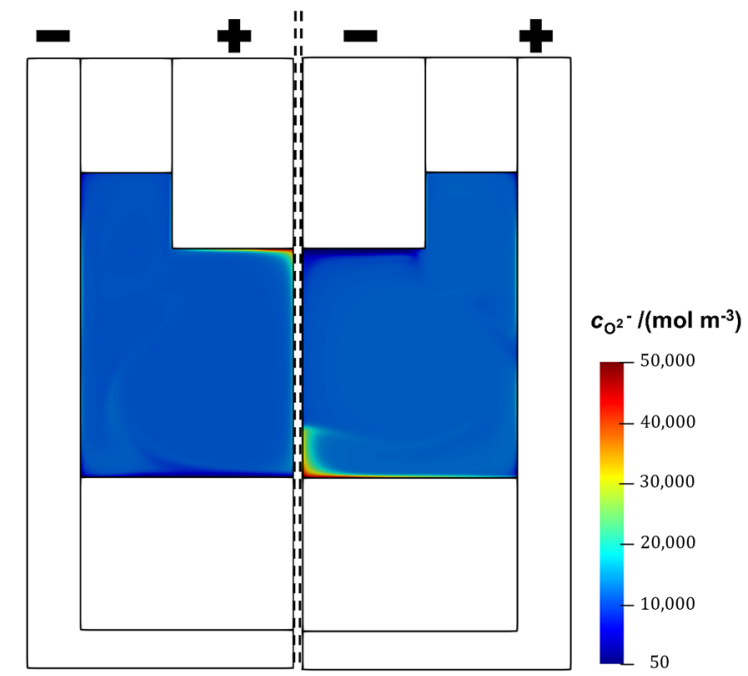

(b)

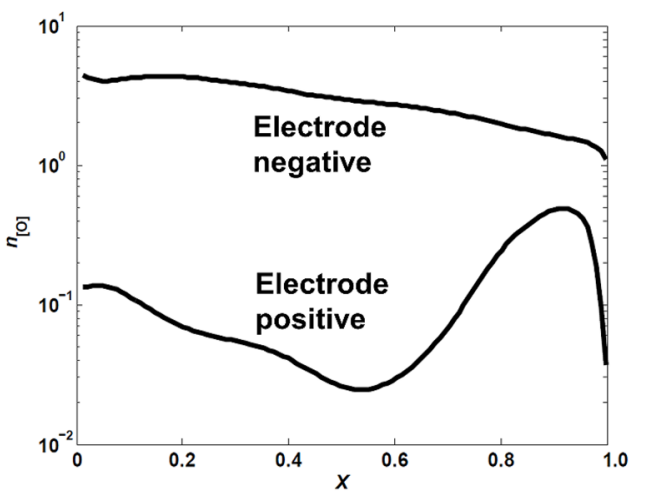

Fig. 7 Concentration field of oxygen ion (a) and normalized amount of precipitated oxygen across slag-melt interface (b) 
magnitude higher than that using DCRP. The concentration of oxygen smoothly decreases from the center of the ingot toward the wall considering DCSP. Contrastingly, the peak value of oxygen concentration is observed near the mold wall (surface of ingot). A relatively high amount of oxygen is also observed near the center.

The presented model in this study was successfully verified [11, 12, 21, 31]. However, the quantitative measurements of oxygen content along the ingot surface have not been reported due to difficulty in measurements at elevated temperature $(\sim 2000 \mathrm{~K})$ and opacity of materials during the ESR process. Nevertheless, this study helps us shed light on the role of electrochemical effects on MHD in slag/metal and the refining mechanism, especially for the oxygen during DC-operated ESR. Herein, we proposed an explanation for a phenomenon, namely higher oxygen content in the final ingot for the ESR process using DCSP than that using DCRP.

\section{Conclusions}

The polarity of the electrode and the modeling approach (tertiary or primary) significantly influence the electric current density field and consequently the electro-vortex flow field in ESR. The calculated toroidal flow in the melt pool is minimal considering the ohmic approach.

Anions $\left(\mathrm{F}^{-}, \mathrm{O}^{2-}\right)$ can reach and accumulate near the anode (either electrode or slag-melt interface depending on the polarity of electrode). The electro-migration can surpass the advection of anions for the anodic electrode as known as DCRP. The electro-migration is assisted by electro-vortex flow for the cathodic electrode as known as DCSP.

Unexpectedly, an enriched region of cation near the anode (either electrode or slag-melt interface depending on the polarity of electrode) is observed due to an enormous injection of $\mathrm{Fe}^{2+}$ into the slag. Despite a relatively uniform concentration of ions in the bulk of slag, the peak of calculated electrical conductivity exists near the vortex where temperature and subsequently the diffusion coefficients reach their peak values. Eventually, the modeling results enable us to put forward an explanation for the experimental observation of higher oxygen content in the ESR ingot using DCSP than that using DCRP.

Acknowledgements The authors acknowledge financial support from the Austrian Federal Ministry of Economy, Family and Youth and the National Foundation for Research, Technology and Development within the framework of the Christian-Doppler Laboratory for Metallurgical Applications of Magnetohydrodynamics.

Open Access This article is licensed under a Creative Commons Attribution 4.0 International License, which permits use, sharing, adaptation, distribution and reproduction in any medium or format, as long as you give appropriate credit to the original author(s) and the source, provide a link to the Creative Commons licence, and indicate if changes were made. The images or other third party material in this article are included in the article's Creative Commons licence, unless indicated otherwise in a credit line to the material. If material is not included in the article's Creative Commons licence and your intended use is not permitted by statutory regulation or exceeds the permitted use, you will need to obtain permission directly from the copyright holder. To view a copy of this licence, visit http://creativecommons. org/licenses/by/4.0/.

Funding Open access funding provided by Montanuniversität Leoben.

\section{References}

[1] G. Hoyle, Electroslag processes, Applied Science Publishers, Essex, England, 1983.

[2] K. Fezi, J. Yanke, M.J.M. Krane, Metall. Mater. Trans. B 46 (2015) 766-779.

[3] A. Jardy, D. Ablitzer, Mater. Sci. Technol. 25 (2009) 163-169.

[4] K.O. Yu, J.A. Domingue, G.E. Maurer, H.D. Flanders, JOM 38 (1986) 46-50.

[5] A. Mitchell, Ironmak. Steelmak. 1 (1974) 172-179.

[6] D.A.R. Kay, R.J. Pomfret, J. Iron Steel Inst. 12 (1971) 962-965.

[7] M.E. Peover, J. Inst. Metals 100 (1972) 97-106.

[8] M. Etienne, The loss of reactive elements during electroslag processing of iron-based alloys, University of British Columbia, Vancouver, Canada, 1971.

[9] L.Z. Chang, X.F. Shi, H.S. Yang, Z.B. Zheng, J. Iron Steel Res. Int. 16 (2009) No. 4, 7-11.

[10] M. Kawakami, K. Nagata, M. Yamamura, N. Sakata, Y. Miyashita, K.S. Goto, Testsu-to-Hagane 63 (1977) 2162-2171.

[11] E. Karimi-Sibaki, A. Kharicha, M. Wu, A. Ludwig, J. Bohacek, Steel Res. Int. 88 (2017) 1700011.

[12] E. Karimi-Sibaki, A. Kharicha, M. Wu, A. Ludwig, J. Bohacek, Ionics 24 (2018) 2157-2165.

[13] M. Kato, K. Hasegawa, S. Nomura, M. Inouye, Trans. ISIJ 23 (1983) 618-627.

[14] G.T. Beynon, Electrochemical aspects of D.C. electroslag remelting, University of British Columbia, Vancouver, Canada, 1967.

[15] Y. Kojima, M. Kato, T. Toyoda, M. Inouye, Trans. ISIJ 15 (1975) 397-406.

[16] A. Mitchell, G.T. Beynon, Metall. Trans. 2 (1971) 3333-3345.

[17] M.E. Fraser, A. Mitchell, Ironmak. Steelmak. 5 (1976) 279-287.

[18] X.C. Huang, B.K. Li, Z.Q. Liu, Metall. Mater. Trans. B 49 (2018) 709-722.

[19] Q. Wang, G.Q. Li, Z. He, B.K. Li, Appl. Therm. Eng. 114 (2017) 874-886.

[20] J. Newman, K.E. Thomas-Alyea, Electrochemical systems, John Wiley \& Sons, New Jersey, USA, 2004.

[21] E. Karimi-Sibaki, A. Kharicha, M. Wu, A. Ludwig, J. Bohacek, J. Electrochem. Soc. 165 (2018) E604-E615.

[22] M. Rosales, J.L. Nava, J. Electrochem. Soc. 164 (2017) E3345E3353.

[23] A. Kharicha, E. Karimi-Sibaki, M. Wu, A. Ludwig, J. Bohacek, Steel Res. Int. 89 (2018) 1700100.

[24] M. Allibert, H. Gaye, J. Geiseler, D. Janke, B.J. Keene, D. Kirner, M. Kowalski, J. Lehmann, K.C. Mills, D. Neuschütz, R. Parra, C. Saint-Jours, P.J. Spencer, M. Susa, M. Tmar, E. Woermann, Slag atlas, Verlag Stahleisen GmbH, Düsseldorf, Germany, 1995. 
[25] M. Barati, K.S. Coley, Metall. Mater. Trans. B 37 (2006) 51-60.

[26] A. Kharicha, M. Wu, A. Ludwig, E. Karimi-Sibaki, J. Iron Steel Res. Int. 19 (2012) 63-66.

[27] E. Karimi-Sibaki, A. Kharicha, M. Wu, A. Ludwig, J. Bohacek, H. Holzgruber, B. Ofner, A. Scheriau, M. Kubin, Appl. Therm. Eng. 130 (2018) 1062-1069.

[28] E. Karimi-Sibaki, A. Kharicha, J. Bohacek, M. Wu, A. Ludwig, Metall. Mater. Trans. B 46 (2015) 2049-2061.

[29] K. Preis, I. Bardi, O. Biro, C. Magele, W. Renhart, K.R. Richter, G. Vrisk, IEEE Trans. Magn. 27 (1991) 3798-3803.
[30] H. Song, N. Ida, IEEE Trans. Magn. 27 (1991) 4012-4015.

[31] E. Karimi-Sibaki, A. Kharicha, M. Wu, A. Ludwig, J. Bohacek, Metall. Mater. Trans. B 51 (2020) 871-879.

[32] H. Versteeg, W. Malalasekera, An introduction to computational fluid dynamics: the finite volume method, Pearson Education Limited, Essex, UK, 2007. 